\title{
Sensoristic approach to biological damage and risk assessment
}

\author{
L. Campanella \& C. Costanza \\ Department of Chemistry, University of Rome "La Sapienza", \\ Rome, Italy
}

\begin{abstract}
A review is proposed of various types of sensoristic approaches to biological damage and risk assessment. The traditional approach to the toxicity evaluation of a matrix based on the determination of toxic concentration is replaced by a sensoristic one. Some sensors that are able to detect risky and dangerous situations and to evaluate them by screening and marker procedures are presented. The marker indexes we propose are total organic carbon, total radicalic concentration, integral toxicity and ecopermanence.

Keywords: TOC analysis, ecopermanence, $\mathrm{TiO}_{2}$ photosensor, biosensor, integral toxicity assessment, human tissue biosensor.
\end{abstract}

\section{Introduction}

There are a continuously increasing number of compounds, both newly discovered and already known, which are disposed of into the environment and then pass into the food chain. Very complicated controls and analysis are required to ascertain whether there are any risks to citizens and users.

These analyses are generally very costly so they are not always completed and if they are completed, it may not be as frequently as is necessary - and they are not permitted everywhere. It is also to be observed that sometimes the obtained results show that the fear of pollution of the considered matrix is disproportionate, as the pollution level is insignificant and below dangerous levels, so that analysis could have been avoided, but obviously this cannot be foreseen.

So a new approach must be more opportunely adopted: the approach of the "crossing light", preliminary screening and markers. The first step of this 
approach is a simple test which entails waiting for a red or green light that means a negative or a positive sample: if it is green, a detailed and specific analysis can be avoided; if it is red, analyses must be carefully performed in order to find the cause of the negative indication. These analyses can be carried out with a preliminary screening that limits the analysis to samples characterised by values of the key indexes close to the limit values $( \pm 20 \%$ can be considered a reasonable range). Finally, when the screening step shows the need for a more detailed investigation, markers can be adopted giving information and capable of ordering the positive samples according to their toxicity. The choice of these markers depends on the type of matrix, but the research advancements able to be dynamically considered individuate continuously new markers for the fundamental role of integral parameters. Finally when the marker test also confirms the danger and the risk of significant toxic levels a complete full analysis has to be performed, generally by hyphenate methods such as GC-MS, HPLC - MS, CE - MS, LCP - MS.

In this paper we want to propose some new instruments for the first steps of the suggested approach. Therefore some biosensors that are able to detect risky and dangerous situations (red crossing light) and to evaluate them by screening and marker procedures are presented. The markers we propose are total organic carbon, total radicalic concentration, integral toxicity and ecopermanence.

The sensors are tools substantially born for analytical aims, the most interesting characteristics of which are the simple operating procedure, the rapidity, the contained costs, the possibility of use in flowing systems, both in situ and with automatic engines.

The analysis of inorganic compounds received particular attention from the scientific community whilst with the organic compounds, the sensor is very often coupled with a biological system, so that a biosensor is being produced. This biological system (enzyme, tissue, micro-organism, whole cells) is sometime immobilised in a membrane. On passing from synthetic to real samples, problems can sometimes arise, as these can contain compounds that are able to deactivate the biological systems (for instance due to enzyme denaturation). The use of biosensors is continuously increasing, especially in the fields of food, chemical, biological, environmental analysis.

\section{$1.1 \mathrm{TiO}_{2}$ sensors for TOC (Total Organic Carbon) analysis}

TOC is an instrumental index, the determination of which consists of the catalytic oxidation of total organic carbon to $\mathrm{CO}_{2}$, the produced amount of which is assumed to calculate the TOC index. TOC is a primary marker of the healthy or unhealthy state of an ecosystem and a very widely-used datum for monitoring organic pollutants, both natural and synthetic.

Luckily, most of the former are not noxious to humans, but the latter, on the contrary, are generally heavily involved in problems of safety and hygiene. Generally, national or international laws do not give any limit value due to the fact that the same TOC values have different significance according to the compounds accounting for them. So in many cases the profile is more relevant within the time scale: sharp upward variations are surely to be feared and 
contrasted. The industrial analysers are based on combustion at high temperatures or on the coupling between a UV reactor and persulphate treatment with conductimetric or infrared determination of produced carbon dioxide.

There is an increasing demand for the measurement of organic carbon in solutions in both industry and environmental research for the purpose of continuous water quality monitoring. Practically all the methods used are based on a catalytic system in which a metal oxide plays a major role. The development of the new TOC measurement system we performed uses a mixed anatase-rutile form $\mathrm{TiO}_{2}$ as a catalyst to the photomineralisation process, and direct measurement of the $\mathrm{CO}_{2}$ produced, by a gaseous diffusion electrode. Titanium dioxide is a well-known photocatalyst. Less well-known are its characteristics as a sensor material. In this paper we describe some recent experimental results concerning the adoption of $\mathrm{TiO} 2$ (in its prevailing anatase form). The research is based on heterogeneous catalysis using an immobilised catalyst, which can offer a considerable advantage over other methods of catalysis.

Four catalysts were tested [1], all based on titanium dioxide in its mixed rutile-anatase form. In three cases, $\mathrm{TiO}_{2}$ was used in the $\mathrm{P} 25$ nanoparticled form produced by Degussa, often described in literature [1] as an excellent catalyst for photodegradation. Most of the oxide was in the crystalline anatase form. It was tested both supported on a glass or metal grid and suspended in solution. In one of the tested catalysts, the titanium grid was oxidised directly in order to enhance $\mathrm{TiO}_{2}$ adhesion to the metal support and to obtain a more uniform surface distribution. A highly pure submillimetric titanium grid with suitable mechanical properties was supplied by Delker (CT, USA) [2].

According to the chemometric treatment of the results using PCA (principal component analysis), the catalytic efficiency scale we obtained confirms these expectations and despite the compounds used, it nevertheless demonstrates the superiority of $\mathrm{TiO}_{2}$ in suspension. Therefore, $\mathrm{TiO}_{2}$ in the form of anatase nanoparticled suspension was used, in the presence of a phosphate buffer, which was unable to interfere with the TOC value, and operating in such a dimension model that enabled the acquisition of a portable TOC monitor. The cell is Plexiglas and the UV radiation corresponds to $350 \mathrm{~nm}$. An auxiliary cell allows a good mixture. GC-MS was applied to determine intermediates and finally check the complete photomineralisation of ions in the organic compounds.

\section{$1.2 \mathrm{TiO}_{2}$ photosensor of the ability of a compound to be mineralised and of environment assessment}

The difference between degradation and mineralization must be emphasised. The former brings a compound to a lower molecular weight, the toxicity of which can also be higher than that of the original compound, and the latter ensuring the total production of $\mathrm{CO}_{2}, \mathrm{H}_{2} \mathrm{O}$ and the mineral acids corresponding to the elements present in the mineralized molecule. The potential for the pollutants to be degraded down to mineralization is an important characteristic for the protection of the environment. To evaluate this can be useful for environmental quality assessment. 


\subsection{1 $\mathrm{TiO}_{2}$ as a sensor of the potential for a compound to be mineralised}

During mineralization, acidification occurs. $\mathrm{TiO}_{2}$ is a photocatalyst of the process [3], but also behaves as a $\mathrm{pH}$ sensor [4] so, at the same time as activating a process, it is also possible to monitor proceedings. The time needed in order to record a $\mathrm{pH}$ shift to more acidic values (or a potential shift to more positive values) can be assumed as a delay proportional to the time of recalcitrance of the tested compound (Figure 1).

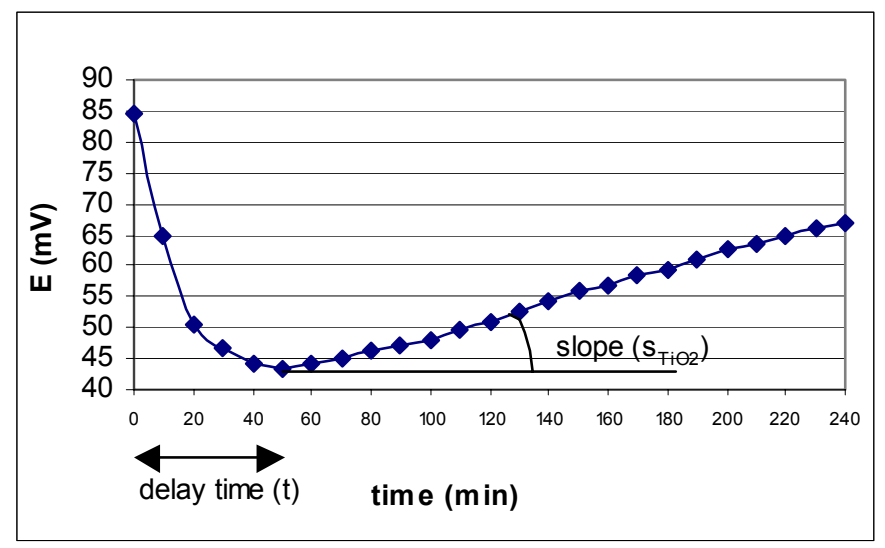

Figure 1: Curve $\mathrm{E}=\mathrm{f}(\mathrm{t}), \mathrm{E}=\mathrm{TiO}_{2}$ potential; $\mathrm{t}=$ irradiation time.

Table 1: Delay time and slope of $\mathrm{pH}$ decrease (potential $\mathrm{E}_{\mathrm{TiO} 2}$ increase) vs. time of some common pollutants.

\begin{tabular}{|c|c|c|}
\hline Compound and concentration & $\begin{array}{l}\text { Delay Time }(\mathrm{min}) \\
(\mathrm{RSD} \%= \pm 6)\end{array}$ & Slope $(\Delta \mathrm{pH} / \Delta \mathrm{min})$ \\
\hline $\mathrm{p}$-chlorophenol $10^{-2} \mathrm{~mol} / \mathrm{L}$ & 30 & 0.15 \\
\hline m-chlorophenol $10^{-2} \mathrm{~mol} / \mathrm{L}$ & 30 & 0.31 \\
\hline o-chlorophenol $10^{-2} \mathrm{~mol} / \mathrm{L}$ & 60 & 0.22 \\
\hline Hydroquinone $10^{-2} \mathrm{~mol} / \mathrm{L}$ & 50 & 0.08 \\
\hline p-quinone $10^{-2} \mathrm{~mol} / \mathrm{L}$ & 20 & 0.04 \\
\hline Glucose $10^{-2} \mathrm{~mol} / \mathrm{L}$ & 60 & 0.04 \\
\hline Carbaryl $10^{-2} \mathrm{~mol} / \mathrm{L}$ & 50 & 0.12 \\
\hline
\end{tabular}

These values agree with other molecular properties related to the stability of the considered compounds.

Further information was obtained by the slope of the $\mathrm{pH}$ decrease following the beginning of the mineralization process denounced by the acidification after the delay time. The obtained values are also shown in the last column of Table 1. It can be tentatively assumed that the ratio between the values of the second column and the corresponding last column are an index accounting for the environmental permanence of a compound, that we can reasonably assume to be inversely proportional to mineralizability.

This parameter could be of great help in the case of an unknown or noncharacterised compound: a White Book of the European Community invites the scientific community to make the most of efforts, in order to set up chemical 
tests that are able to give information - especially alarm advice - in real time (or almost real time) about the toxicity of a compound, the state of a workplace or an environment.

\subsection{Biosensors to evaluate free radical scavenger properties}

Radical reactions are involved in the processes leading to the breakdown of the lipids contained in most foodstuffs and to their rapid deterioration. They are responsible for ageing processes and cause numerous diseases from which considerable economic loss and potential health problems may follow.

As a consequence of the increasing number of biochemical problems involving free radicals, the use of scavenging and antioxidant compounds in the fields of health, drugs and food has grown. Different screening tests have been developed to determine the antioxidant properties of natural and synthetic antioxidant compounds.

To this end, several studies have been reported involving organic solvents, micelles and liposomes, sometimes using the rate of oxygen uptake via the pressure transducer method, or measures by an oxygen electrode. Successively, methods based on conjugated diene formation, which have a higher sensitivity than oxygen uptake and allow spectrophotometric or fluorimetric measurements are proposed, such as RANSOD, in which the superoxide radical reacts with a derivative of phenyltetrazolium chloride to form a red formazan dye, or those that measure the absorbance of the reduced nitro blue tetrazolium compound. Several in vitro tests have been recently proposed for measuring the antioxidant properties of food products, based on the inhibition of human low-density lipoprotein oxidation, or by using the oxygen radical absorbance capacity (ORAC), used to determine the total antioxidant activity.

Our research group recently approached this problem, beginning with the determination of oxygen free radicals, in particular superoxide radical [5], and assembling several new kinds of electrochemical sensors and biosensors suitable for this purpose; firstly a voltammetric system based on the detection of reduced cytochrome c; this system was also applied to develop a suitable amperometric carbon paste electrode; secondly two potentiometric sensors (one classical selective membrane sensor and the other a solid state field effect transistor sensor), based on selective membrane entrapping benzylidenephenylnitrone with potentiometric detection. More recently, we studied two different kinds of biosensors to determine superoxide radicals obtained by coupling a transducer consisting of an amperometric gas diffusion electrode for the oxygen, or another amperometric electrode for hydrogen peroxide, with superoxide dismutase enzyme immobilized in kappa carrageenan gel. Both the sensors showed a suitable response to the superoxide radical. We think that the second type of biosensor is now mature, both from an engineering and an operative point of view, to check the antioxidant properties of several compounds comparing the response of the biosensor both in the presence and in the absence of the considered scavenger compound.

At this end using the $\mathrm{SOD} / \mathrm{H}_{2} \mathrm{O}_{2}$ biosensor set up in our laboratory, we recently evaluated the scavenging properties in vitro of important molecules such 
as cysteine, melatonin, $\beta$-carotene and acetyl salicylic acid. In addition, we addressed this problem through the study of scavenging properties of fresh vegetal and fruit tissues, or extracts of the same fruits and vegetables present on the market and available in chemists' or herbalists' shops; lastly, the investigation was extended to include several specific active principles contained in the examined vegetal tissues.

We also addressed the problem of the experimental evaluation of any difference in the defence against free radicals (that is, in practice, the antiradical properties) of human kidney tissues both healthy and diseased, following a completely original approach, starting from our experience in designing and building a superoxide dismutase biosensor. In order to apply it, devoted to superoxide radical determination, radicals must be produced in a quantitative way. So we produced radicals by xanthine oxidation (to uric acid) in the presence of xanthine oxidase and measured the scavenging action of human kidney tissue both from a healthy and a cancerous organ, concluding that the antiradical capacity of the former is much higher than the latter.

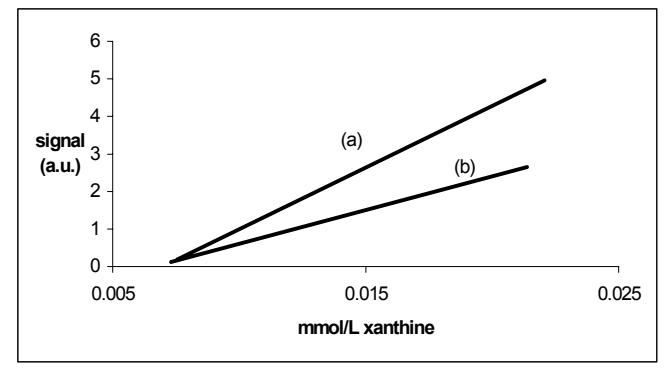

Figure 2: Typical (SOD) biosensor calibration curves, (a) in absence and (b) in presence of antioxidant matrices.

\subsection{Biosensor for integral toxicity assessment}

For some years, the toxicity of many chemical substances has been studied by means of living organisms used as biological indicators. Higher organisms are generally used, such as the guinea-pig, as the results they produce are often extremely reliable, even though the response times may be lengthy (ranging from a few days to several months, or even years in tests for chronic toxicity). Nevertheless, by exploiting unicellular organisms, in particular yeasts, it is possible to reduce the testing time considerably. Furthermore, by using suitable biological systems, such as immobilised yeast colonies, it is possible to implement the method even in-situ, with the advantage of obtaining a value of integral toxicity as due neither to this nor to that compound but to all these together with the eventual antagonisms and synergisms too.

An immobilised yeast cell biosensor $[6,7]$ has been developed for the total toxicity testing of a sample that may contain a number of different pollutant species; the biosensor uses amperometric gas diffusion oxygen as an indicator electrode. The method is based on the perturbation of the respiratory activity of a yeast, Saccharomyces cerevisiae, immobilised on an agar medium containing the 
culture medium (i.e., 'agarised medium'), due to the toxic tested substance. Glucose is used as a substrate while the tested species consisted of several metallic ions, phenols, cationic and anionic surfactants, pesticides and other toxics.

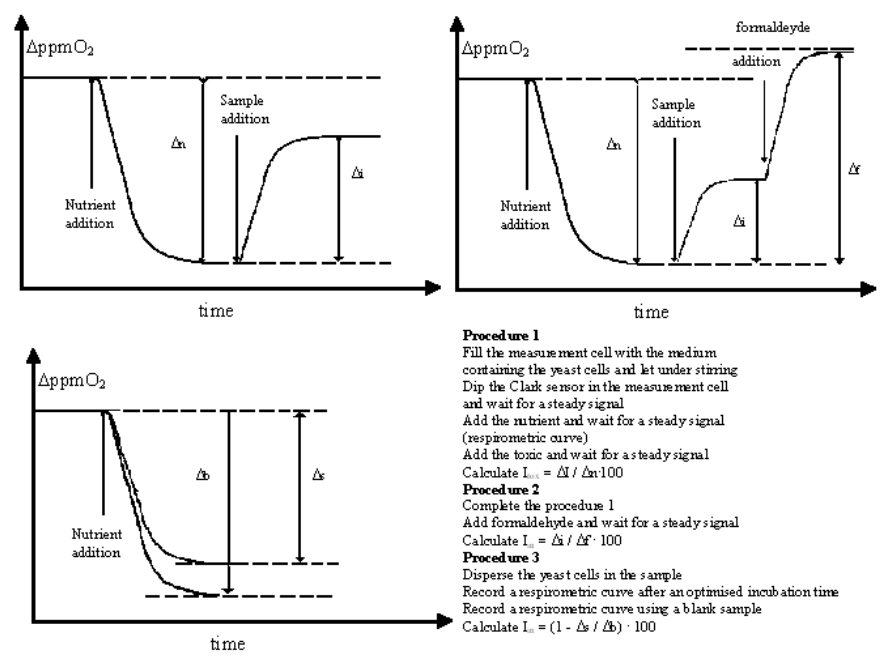

Figure 3: Various respirometric curves recorded in both normal conditions and after exposure of the system to the tested toxic substance.

The measurements are performed by allowing the biosensor to stabilize under magnetic stirring, in the glass cell thermostated at $25^{\circ} \mathrm{C}$, containing a fixed electrolytic solution $(15 \mathrm{ml})$, isotonic with the yeast cell plasma. The next step is to add a standard glucose solution (final concentration $0.1 \% \mathrm{w} / \mathrm{v}$ ), and the decrease of dissolved oxygen concentration caused by the increase in the yeast cell respiration process is recorded as a function of time; in practice, we obtained what is commonly called the 'respirometric curve', which shows the behaviour of oxygen consumption as a function of time. From an examination of the various respirometric curves recorded in both normal conditions and after exposure to the system of toxic substances at different concentrations, we obtained a calibration curve to refer in order to determine the toxicity of the selected sample. In practical terms, for each measurement, two different respirometric curves are recorded: one in the presence of the substrate alone and the other in the presence of the substrate but after an initial incubation with the toxic substance $(8 \mathrm{~min}$.); the results can by processed using one of the two following sets of experimental data (Figure 3):

a) difference in final current intensity $(\delta)$, which is proportional to the 'distance' between the respirometric curves, at a fixed time (30 min.) after the addition of the glucose;

b) the difference in amplitude of the angles $\left(\alpha-\alpha_{0}\right)$ referring to the slopes by which the two respirometric curves attain a stationary state, which is measured $25 \mathrm{~min}$ after the addition of glucose. 
In this way it is possible to monitor integral toxicity of water from STP, especially if it is destined for drinking water, of soils incidentally polluted and followed during bioremediation of sediments or of industrial media. Two very important applications were performed on radon pollution and on the effects of cellular phones on biological systems, both are the object of controversies due to the contrasting conclusions reached by different authors, very often basing their conclusions on unclear and less accurate measurements. Particularly for cellular phone field it has been concluded that 900 and $1800 \mathrm{MHz}$ cellular phones, corresponding to first and second generation phones, can be considered dangerous if the user is exposed to the effects for longer than 5 hours. In any case, total reversibility was observed after 48 hours since the last exposure.

\subsection{Algal biosensor}

Eukaryotic and prokaryotic microalgae are regarded as relevant indicators in the field of environmental monitoring and risk assessment. As a result of their easiness of culture and sensitivity to a number of pollutants, they are frequently used in ecotoxicological screening of contaminated fresh and seawater. In toxicity tests, several parameters can be measured to assess the effects of toxicants on microscopic algae. Growth and photosynthetic activity are the most commonly monitored factors [8-9].

Many different chemical pollutants have been assayed for their effects on algal species. Particularly, the interactions between microalgae and trace metals have been extensively studied, with reference to both bioaccumulation [10-11] and toxicity [12]. Microalgae are also used as test species in ascertaining the environmental effects of a variety of synthetic chemicals for pesticide use, primarily herbicides, but also insecticides and fungicides [13]. Most of the proposed devices are based on the electrochemical detection of the inhibiting effect on the photosynthetic activity of algae and cyanobacteria exerted by some toxicants.

Algal biosensors may provide a successful solution to the need for automatic devices and alert systems allowing real time detection and on-line monitoring.

Our analytical device is represented by an amperometric biosensor able to monitor oxygen consumption and evolution of respectively respirometric and photosynthetic algal activity, obtained by coupling a suited algal bioreceptor with a Clark electrode. The selected algal species is the marine filamentous cyanobacterium Spirulina subsalsa, which exhibited positive responses in all our preliminary tests and, owing to its morphological features, does not require any immobilization procedure.

Measurements were performed as follows: the sample is allowed to flow in the cell under irradiation cycles (dark/light rate $=1 / 4$ ), a saw-toothed signal is registered corresponding to the succession of photosynthesis (light) and respiration (dark) processes of the algae; a random variation not exceeding $5 \%$ can be obtained in the signal height when unpolluted samples are tested (fig. 4a) during at least 48 hours whilst, in the presence of added pollutants, it decreases as shown in fig. $4 \mathrm{~b}$; the time $\left(\mathrm{t}_{\mathrm{l} / 2}\right)$ required to reduce the height to a half can be related to the toxicity (and of course concentration) of the considered pollutant. 
(a)

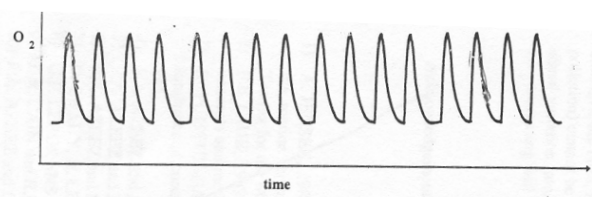

(b)

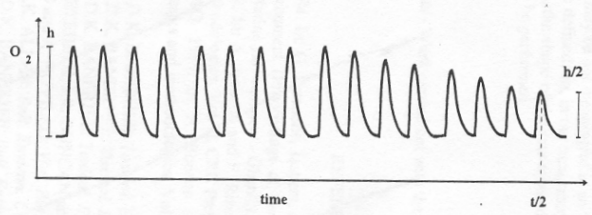

Figure 4: Experimental plot obtained in the analysis of blank (a) and sample (b).

Table 2: Data obtained using algal biosensor in the analysis of organic pollutants.

\begin{tabular}{|c|c|c|c|c|c|c|c|}
\hline Pollutant & Concentration & $\begin{array}{l}\mathbf{t}_{1 / 2} \\
(\min )\end{array}$ & $\begin{array}{l}\text { RSD } \\
\%\end{array}$ & Pollutant & Concentration & $\begin{array}{l}\mathbf{t}_{1 / 2} \\
(\min )\end{array}$ & \begin{tabular}{|l|} 
RSD \\
$\%$
\end{tabular} \\
\hline \multirow{4}{*}{ Atrazine } & $20 \mu \mathrm{g} / \mathrm{L}$ & 690 & 5.8 & \multirow{4}{*}{$\begin{array}{l}\text { p- } \\
\text { chlorophenol }\end{array}$} & $1 \cdot 10^{-4} \mathrm{~mol} / \mathrm{L}$ & 1080 & 0.5 \\
\hline & $50 "$ & 290 & 8.6 & & $1 \cdot 10^{-3} "$ & 312 & 2.9 \\
\hline & $100 "$ & 130 & 7.7 & & $1 \cdot 10^{-2} "$ & 89 & 11 \\
\hline & $150 "$ & 90 & 4.4 & & & & \\
\hline \multirow{4}{*}{ Terbutilazine } & $0.5 \mu \mathrm{g} / \mathrm{L}$ & 76 & 6.5 & \multirow{4}{*}{$\begin{array}{l}\text { Trifluoracetic } \\
\text { acid }\end{array}$} & $1 \cdot 10^{-4} \mathrm{~mol} / \mathrm{L}$ & 528 & 0.7 \\
\hline & $0.5 "$ & 48 & 14 & & $1 \cdot 10^{-3} "$ & 147 & 4.8 \\
\hline & $1.0 "$ & 42 & 7.1 & & $1 \cdot 10^{-2}$ & 91 & 7.7 \\
\hline & $2.5 "$ & 51 & 11 & & & & \\
\hline \multirow{5}{*}{ Parationmetil } & $1.7 \mathrm{mg} / \mathrm{L}$ & 817 & 0.1 & \multirow{5}{*}{$\begin{array}{l}\text { Banzalkonium } \\
\text { chloride }\end{array}$} & $1 \cdot 10^{-4} \mathrm{~mol} / \mathrm{L}$ & 507 & 2.2 \\
\hline & $2.5^{\prime \prime}$ & 554 & 1.0 & & $1 \cdot 10^{-3} "$ & 188 & 3.7 \\
\hline & $3.5 "$ & 325 & 138 & & $1 \cdot 10^{-2} "$ & 161 & 0.6 \\
\hline & $4.0 "$ & 310 & 170 & & & & \\
\hline & $5.5 \mathrm{~m}$ & 250 & 240 & & & & \\
\hline \multirow{4}{*}{ Carbaryl } & $0.5 \mathrm{mg} / \mathrm{L}$ & 740 & 3.9 & \multirow{4}{*}{ Tween 80} & $1 \cdot 10^{-3} \mathrm{~mol} / \mathrm{L}$ & 463 & 1.1 \\
\hline & $1.0 \mathrm{"}$ & 370 & 5.4 & & $5 \cdot 10^{-3} "$ & 415 & 0.7 \\
\hline & $2.5 "$ & 155 & 6.5 & & $1 \cdot 10^{-2} "$ & 399 & 1.7 \\
\hline & $5.0^{\prime \prime}$ & 70 & 7.1 & & & & \\
\hline
\end{tabular}

Table 2 lists all the obtained results. It can be seen that, as expected, terbutilazine and atrazine were the most toxic compounds respectively (shorter half time at lower concentration); moreover, terbutilazine toxicity remains higher than some of the other considered pollutants that, in turn, result in being more toxic than the other compounds, which means that synergetic and antagonist effects occur. The results show the suitability of the proposed biosensor in the evaluation of sea water quality; today its use can be suggested for the integral toxicity tests of estuaries and bays that are close to doubtful quality matrices such as industrial or civil effluents. It has to be pointed out that the parameter $\mathrm{t}_{1 / 2}$ (time to reach half height of the saw-toothed signal), even if widely used in this kind of test and for this reason, can today be adopted by us in order to compare our results with bibliographic ones, may be not meaningful in an "in situ" analysis where a zero time (starting time of the pollution) cannot be defined. 
Therefore in our next research, alternative indexes will be looked for, i.e. the signal variation rate, the shape of the saw-toothed signal and of the whole plot. Chemiometric data processing will also be performed.

\section{References}

[1] Campanella L., De Angelis G, Visco G., Chemometric investigation of efficiency response of different $\mathrm{TiO}_{2}$ based catalysts as principal component of TOC photochemical sensor. Anal. Bioanal. Chem., 376, pp.467-475, 2003.

[2] DELKER Corporation 14 Commercial Street - PO Box 427 - Branford, CT 06405, www.delkergrid.com

[3] Mills A., Le Hunte S., An overview of semiconductor photocatalysis. $J$. Photochem. Photobiol. A: Chem., 108, pp. 1-352, 1997.

[4] Campanella L., Battilotti M., Costanza C., Studies on simulated ageing of paper by photochemical degradation. Ann. Chim, 95, 2005, pp. 727-740.

[5] Campanella L., Favero G., Tomassetti G., Superoxide dismutase biosensors for superoxide radical analysis. Anal. Lett, 32, pp. 2559-81, 1999.

[6] Campanella L., Favero G., Mastrofini D., Tomassetti G., Further developments in toxicity cell biosensors. Sens. Act. B Chem., 44, pp. 279285, 1997.

[7] Campanella L., Cubadda F, Sammartino M.P, Tonnina D., Visco G., Integral toxicity test of sea waters by algal biosensor.Ann.Chim.92pp.477484, 2002.

[8] Jensen A., Marine ecotoxicological tests with phyto-plankton. Proc. of the International Symposium on Ecotoxicological Testing for the Marine Environment-Ghent, Belgium, 12-14 September 1983, eds G. Persoone, E. Jaspers, C. Claus, pp. 195-213, 1984.

[9] Puiseux-Dao S., "Phytoplankton model" in ecotoxicology. Aquatic Ecotoxicoology: Fundamental Concepts and Methodologies, Vol. II, eds A. cology. A. Boudou and F. Ribeyre, pp. 163-185. CRC Press, lnc., Boca Raton, Florida, 1989.

[10] Azeez P. \& Banerjee D., Effect or copper and cadmium on carbon assimilation and uptake or metals by algae. Toxic. Environ. Chem., 12, pp. 77-86, 1986.

[11] Azeez P. A. and Banerjee D. K., Effect of chromium on cyanobacteria and its accumulation. Toxic. Environ. Chem., 16, pp. 229-240, 1988.

[12] Cain J. R. \& Allen R. K., Use of a cell wall-less mutant strain to assess the role of the cell wall in cadmium and mercury tolerance by Chlamydomonas reinhardtii. Bull. Environ. Contam. Toxic. 25, pp.797 801, 1980.

[13] Padhy R., Cyanobacteria and pesticides. Residue Reviews, 95, pp.1-44, 1985. 